\title{
Mean Template for Tensor-Based Morphometry Using Deformation Tensors
}

\author{
Natasha Leporé ${ }^{1}$, Caroline Brun ${ }^{1}$, Xavier Pennec ${ }^{2}$, Yi-Yu Chou ${ }^{1}$, \\ Oscar L. Lopez ${ }^{3}$, Howard J. Aizenstein ${ }^{4}$, James T. Becker ${ }^{4}$, Arthur W. Toga ${ }^{1}$, \\ and Paul M. Thompson ${ }^{1}$ \\ ${ }^{1}$ Laboratory of Neuro Imaging, UCLA, Los Angeles, CA 90095, USA \\ ${ }^{2}$ Asclepios Research Project, INRIA Sophia-Antipolis, 2004 route des Lucioles 06902 \\ Sophia-Antipolis Cedex, France \\ ${ }^{3}$ Department of Psychiatry, University of Pittsburgh, Pittsburgh, PA 15213 USA \\ ${ }^{4}$ Department of Neurology, University of Pittsburgh, Pittsburgh, PA 15213 USA
}

\begin{abstract}
Tensor-based morphometry (TBM) studies anatomical differences between brain images statistically, to identify regions that differ between groups, over time, or correlate with cognitive or clinical measures. Using a nonlinear registration algorithm, all images are mapped to a common space, and statistics are most commonly performed on the Jacobian determinant (local expansion factor) of the deformation fields. In [14, it was shown that the detection sensitivity of the standard TBM approach could be increased by using the full deformation tensors in a multivariate statistical analysis. Here we set out to improve the common space itself, by choosing the shape that minimizes a natural metric on the deformation tensors from that space to the population of control subjects. This method avoids statistical bias and should ease nonlinear registration of new subjects data to a template that is 'closest' to all subjects' anatomies. As deformation tensors are symmetric positive-definite matrices and do not form a vector space, all computations are performed in the log-Euclidean framework [1. The control brain $B$ that is already the closest to 'average' is found. A gradient descent algorithm is then used to perform the minimization that iteratively deforms this template and obtains the mean shape.

We apply our method to map the profile of anatomical differences in a dataset of $26 \mathrm{HIV} /$ AIDS patients and 14 controls, via a log-Euclidean Hotelling's $T^{2}$ test on the deformation tensors. These results are compared to the ones found using the 'best' control, $B$. Statistics on both shapes are evaluated using cumulative distribution functions of the $p$ values in maps of inter-group differences.
\end{abstract}

\section{Introduction}

Tensor-based morphometry (TBM) is an increasingly popular method to study differences in brain anatomy statistically [23, 5, 22. In TBM, a non-linear registration algorithm is used to align a set of images to a common space, and a statistical analysis is typically performed on the Jacobian determinants (local 
expansion factors) of the deformation fields generating the transformation. Most commonly, one of the control subjects' images, or a high-resolution single subject MRI atlas [10], is selected as the reference to which all the other images are mapped. To avoid biases induced by the choosing a single individual as a template, methods for creating an average image using the entire set of controls have also been developed. For instance in [11, a mean template is defined by transforming one of the control images using the average of the displacement fields resulting from its registration to all other controls. A similar approach was also adopted in [9], where the average was taken with respect to both the deformation and the intensities of the reference images.

Other investigators have advocated a more computationally intensive 'targetless' normalization approach, in which all images in a group are matched to each other pairwise, and each image's mean vector field so obtained is applied to it before averaging the deformed images across subjects [24], 22], 28], 27], [12]. Groupwise registration is increasingly common to avoid systematic confounding effects and bias associated with aligning images to a specific individual brain, which can arise when the geometry and intensities of the target image resemble some members of the population more than others.

In [14, the deformation tensors $\sqrt{J^{t} J}$ were used to perform statistics in TBM, where $J$ is the local Jacobian matrix of the transformation. This method outperformed $\operatorname{det} J$, the most commonly used scalar measure of deformation, for mapping the profile of brain atrophy associated with HIV/AIDS. Specifically, multivariate analysis of the local tensor, using a manifold version of the Hotelling $T^{2}$ test, was much more sensitive to group differences than det $J$. The determinants represent local volume differences across subjects, while the deformation tensors reflect local differences in shape, orientation, and volume.

When statistics are performed on the deformation tensors in TBM, a consistent way to define the average image is as one that minimizes and appropriate norm on the deformation tensors generated using that image as a registration target. For example, when a set of control subjects' images is mapped to a template, it is reasonable to expect the mean deformation tensor to be identically zero everywhere after log transformation, or, if that is not possible, at least having minimum mean-squared error in a relevant tensor norm. Here we develop an approach to achieve this, by using a log-Euclidean metric on the space of tensors; the regularizer then has a form that is consistent with the tensor statistics used ultimately for mapping systematic effects on anatomy.

In related work on geodesic shooting [17] and large-deformation diffeomorphic metric mapping (LDDMM) 2], mean templates are defined that minimize the geodesic distance to a population of anatomies. These geodesic distances are Riemannian metrics formulated in terms of integrals of $\|L v\|$, where $L$ is a self-adjoint (elliptic) differential operator regularizing the deformation, $v$ is its velocity field, and $\|$.$\| is a norm, such as the simple L^{2}$ norm or the $H_{\alpha}^{1}$ norm used in the Camassa-Holm equation for modeling solitons [29]. Lorenzen et al. [15] [16] generated a representative common template from a multimodality image set using large-deformation mappings and registration with the Kullback-Leibler 
divergence. Gerig et al. [7] generalized the mean anatomical template estimation to accommodate repeated measures data, e.g. images collected longitudinally from a pediatric population.

In this work, we set out to find a transformation $\Phi_{B A}$ from an initial brain $B$ selected from a set of control subjects, to an average brain $A$. The average brain image intensity is defined as $I_{A}=I_{B} \circ \Phi_{B A} . B$ is taken as the reference image, and we seek the transformation of its geometry that minimizes the bias on the deformation tensors:

$$
\operatorname{argmin}_{\Phi_{B A}} E\left(\Phi_{B A}\right)
$$

where $E\left(\Phi_{B A}\right)$ is the total size of the deformation tensors

$$
E=\sum_{i} \int d\left(S_{i}, I d\right)^{2}
$$

Here the $S_{i}$ represent the square of the deformation tensors from image $i, d(.,$. is the distance, and $I d$ is the identity. In practice, to make calculations easier, we actually compute the inverse transformation, $\Phi_{A B}$. (Note that this formulation could be extended to consider intensity matching as well, as in [15] [16] where the sum of an intensity matching energy and a deformation energy is minimized).

The deformation tensors are constrained to be positive-definite matrices, and form a conical submanifold of the space of square matrices. An intrinsic definition of $d(.,$.$) is needed for (2). Recently, Arsigny et al. [1] presented a log-Euclidean$ framework to perform computations in this space. Distances are computed after applying the matrix logarithm transformation, which transports the deformation tensors to the tangent space at the origin, where simple matrix operations can be used. When log transforms are used, even on the scalar Jacobian determinant, several sources of bias are avoided in the resulting statistics (which can lead to skewness and non-zero mean even under the null hypothesis [13]).

This method was used in 14 to compute statistics on the deformation tensors in TBM. In the log-Euclidean framework, the distance between two elements of the space $S_{1}$ and $S_{2}$ is given by

$$
d\left(S_{1}, S_{2}\right)=\left\|\log S_{1}-\log S_{2}\right\|
$$

where $\|$.$\| denotes a norm, and \log$ is the matrix logarithm. Here we will use [1]

$$
d\left(S_{1}, S_{2}\right)=\left(\operatorname{Trace}\left(\log S_{1}-\log S_{2}\right)^{2}\right)^{1 / 2} .
$$

Taking into account (3), (2) becomes

$$
E=\sum_{i} \int\left\|\log S_{i}\right\|^{2} d^{2} x=\sum_{i} \int \operatorname{Tr}\left(\log S_{i}\right)^{2} d^{2} x
$$

that is, the size of the $S_{i}$ given a transformation of the chosen image $\Phi_{A B}$.

We used a fluid registration algorithm [6] to register the images. The code was accelerated using a convolution filter derived from the Green's function of 
the differential operator in the fluid equation [3] [ 8$]. \Phi_{A B}$ was then computed using gradient descent. As our initial brain $B$, we selected the control subject for which (4) was minimal.

In the next section, we describe our gradient descent algorithm. Our method is then applied to perform a TBM analysis of the corpus callosum in a group of 26 AIDS patients and 14 matched controls.

\section{Method}

A gradient descent method in the log-Euclidean framework was outlined in [19] and 20 for the log-Euclidean elasticity. Here we use the general philosophy described in those references. However, a major added complication is that our method requires two consecutive registrations, from $A$ to $B$ and from $B$ to $i$.

The transformation $\Phi_{A i}\left(r_{A}\right)$ from $A$ to image $i$ at point $r_{A}$ is given as a function of the deformation fields $D$ by 11

$$
\Phi_{A i}\left(r_{A}\right)=\Phi_{B i} \circ \Phi_{A B}\left(r_{A}\right)=r_{A}+D_{A B}\left(r_{A}\right)+D_{B i}\left(r_{A}+D_{A B}\left(r_{A}\right)\right)
$$

The value of $S_{i}$ from $A$ to image $i$ is thus given by

$$
S_{i}\left(\Phi_{A B}\right)=\sum_{\alpha} \partial_{\alpha}\left(\Phi_{B i} \circ \Phi_{A B}\right) \partial_{\alpha}\left(\Phi_{B i} \circ \Phi_{A B}\right)^{t} .
$$

Using

$$
\begin{array}{r}
S_{i}\left(\Phi_{A B}+\epsilon u\right)=\sum_{\alpha} \partial_{\alpha}\left(\Phi_{B i} \circ\left(\Phi_{A B}+\epsilon u\right)\right) \partial_{\alpha}\left(\Phi_{B i} \circ\left(\Phi_{A B}+\epsilon u\right)\right)^{t} \\
=\sum_{\alpha} \partial_{\alpha}\left(\Phi_{B i} \circ \Phi_{A B}+\epsilon \sum_{k} u^{k}\left(\partial_{k} \Phi_{B i}\right) \circ \Phi_{A B}+\ldots\right) \\
\partial_{\alpha}\left(\Phi_{B i} \circ \Phi_{A B}+\epsilon \sum_{k} u^{k}\left(\partial_{k} \Phi_{B i}\right) \circ \Phi_{A B}+\ldots\right)^{t},
\end{array}
$$

we find the directional derivative of $S_{i}$ in the direction of the vector field $u$

$$
\begin{aligned}
\partial_{u} S_{i}\left(\Phi_{A B}\right)=\sum_{\alpha} & {\left[\partial_{\alpha}\left(\Phi_{B i} \circ \Phi_{A B}\right)\right]\left[\partial_{\alpha}\left(\sum_{k} u^{k}\left(\partial_{k} \Phi_{B i}\right) \circ \Phi_{A B}\right)\right]^{t} } \\
& +\left[\partial_{\alpha}\left(\sum_{k} u^{k}\left(\partial_{k} \Phi_{B i}\right) \circ \Phi_{A B}\right)\right]\left[\partial_{\alpha}\left(\Phi_{B i} \circ \Phi_{A B}\right)\right]^{t}
\end{aligned}
$$

The directional derivative of the energy gradient for image $i$ is then:

$$
\begin{aligned}
\partial_{u} \int \operatorname{Tr}\left(\log S_{i}\right)^{2} & =2 \int \operatorname{Tr}\left(\log \left(S_{i}\right) S_{i}^{-1} \partial_{u} S_{i}\right) \\
& =4 \int \operatorname{Tr}\left(Z \sum_{\alpha}\left[\partial_{\alpha}\left(\Phi_{B i} \circ \Phi_{A B}\right)\right]\left[\partial_{\alpha}\left(\sum_{k} u^{k}\left(\partial_{k} \Phi_{B i}\right) \circ \Phi_{A B}\right)\right]^{t}\right) \\
& +4 \int \operatorname{Tr}\left(Z \sum_{\alpha}\left[\partial_{\alpha}\left(\sum_{k} u^{k}\left(\partial_{k} \Phi_{B i}\right) \circ \Phi_{A B}\right)\right]\left[\partial_{\alpha}\left(\Phi_{B i} \circ \Phi_{A B}\right)\right]^{t}\right)
\end{aligned}
$$

where $Z \equiv \log \left(S_{i}\right) S_{i}^{-1}$. Integrating by parts, we finally obtain 


$$
\begin{aligned}
\partial_{u} \int \operatorname{Tr}\left(\log S_{i}\right)^{2}= & -4 \int \operatorname{Tr}\left(\left[\sum_{\alpha} \partial_{\alpha}\left(Z \partial_{\alpha}\left(\Phi_{B i} \circ \Phi_{A B}\right)\right)\right]\left[\sum_{k} u^{k}\left(\partial_{k} \Phi_{B i}\right) \circ \Phi_{A B}\right]^{t}\right) \\
& -4 \int \operatorname{Tr}\left(\left[\sum_{k} u^{k}\left(\partial_{k} \Phi_{B i}\right) \circ \Phi_{A B}\right]\left[\sum_{\alpha} \partial_{\alpha}\left(Z \partial_{\alpha}\left(\Phi_{B i} \circ \Phi_{A B}\right)\right)\right]^{t}\right)
\end{aligned}
$$

The total derivative term cancels as the image intensity and thus $\Phi_{A B}$ is zero near enough to the boundary. This can be guaranteed in the general case by padding the image with zeros.

Finally, we obtain the gradient of the energy for image $i$ as

$$
\nabla E_{i}=-4<\sum_{\alpha} \partial_{\alpha}\left(Z \partial_{\alpha}\left(\Phi_{B i} \circ \Phi_{A B}\right)\right) \mid\left(\nabla \Phi_{B i}\right) \circ \Phi_{A B}>.
$$

where $<. \mid .>$ denotes the usual scalar product in $R^{3}$.

\section{$2.1 \quad$ Numerical Implementation}

As an initial condition for the gradient descent, we moved the chosen template $B$ to the location of the average deformation field from $B$ to all other controls. This definition of a 'vector mean' template has been adopted by others [11, but here we optimize it using a further deformation to yield a template with minimal energy in the multivariate log-Euclidean space.

Using a finite difference scheme in the computation of the gradient yields poor results, as a small number of voxels with large gradient values can end up driving the computation, and in such cases most of the image will change very slowly. We remedied this problem using a multi-resolution scheme, for which all derivatives in (5) were computed through convolution with a Gaussian filter, for which the variance was reduced at each resolution step. To improve the speed of convergence, the positions were updated after the computation of the descent direction for each $i$.

\subsection{Data}

Twenty-six HIV/AIDS patients (age: $47.2 \pm 9.8$ years; $25 \mathrm{M} / 1 \mathrm{~F} ; \mathrm{CD}^{+}{ }^{+}$T-cell count: $299.5 \pm 175.7$ per $\mu \mathrm{l}$; $\log _{10}$ viral load: $2.57 \pm 1.28 \mathrm{RNA}$ copies per $\mathrm{ml}$ of blood plasma) and $14 \mathrm{HIV}$-seronegative controls (age: $37.6 \pm 12.2$ years; $8 \mathrm{M} / 6 \mathrm{~F}$ ) underwent 3D T1-weighted MRI scanning; subjects and scans were the same as those analyzed in the cortical thickness study in [25, where more detailed neuropsychiatric data from the subjects is presented. All patients met Center for Disease Control criteria for AIDS, stage C and/or 3 (Center for Disease Control and Prevention, 1992), and none had HIV-associated dementia. All AIDS patients were eligible to participate, but those with a history of recent traumatic brain injury, CNS opportunistic infections, lymphoma, or stroke were excluded.

All patients underwent a detailed neurobehavioral assessment within the 4 weeks before their MRI scan, involving a neurological examination, psychosocial interview, and neuropsychological testing, and were designated as having no, 
mild, or moderate (coded as 0,1 , and 2 respectively) neuropsychological impairment based on a factor analysis of a broad inventory of motor and cognitive tests performed by a neuropsychologist 25 .

All subjects received 3D spoiled gradient echo (SPGR) anatomical brain MRI scans $(256 \times 256 \times 124$ matrix, TR $=25 \mathrm{~ms}, \mathrm{TE}=5 \mathrm{~ms} ; 24-\mathrm{cm}$ field of view; $1.5-\mathrm{mm}$ slices, zero gap; flip angle $=40^{\circ}$ ) as part of a comprehensive neurobehavioral evaluation. The MRI brain scan of each subject was co-registered with a 9parameter transformation to the ICBM53 average brain template, after removal of extracerebral tissues (e.g., scalp, meninges, brainstem and cerebellum).

The corpus callosum of each subject was hand-traced 26, using an interactive segmentation software. The traces were treated as binary objects (1 within the $\mathrm{CC}, 0$ outside), as we wished to evaluate anatomical differences in a setting where intensity was held constant (see Lorenzen et al. 15, 16, where a radiometric term based on information theory was included in the template estimation equations, but tensor statistics were not evaluated).

\section{Results}

The total energy was found to be much lower in the case of the mean template $\left(E_{A}=3.027 \times 10^{3}\right.$ vs $\left.E_{B}=3.794 \times 10^{3}\right) . T^{2}$ statistics identifying group differences in our dataset are shown in Fig. 1a. The cumulative distribution function of the $p$-values is plotted in Fig. $1 \mathrm{~b}$ against the $p$-values that would be expected under the null hypothesis, for both templates. For null distributions (i.e. no group difference detected), these are expected to fall along the $x=y$ line, and larger deviations from that curve represent larger effect sizes. The registration to the average brain gives statistics similar to the one to one individual. Thus we do not sacrifice any of the signal by using our averaging procedure. Furthermore, the average template can be used to remove potential interaction between the
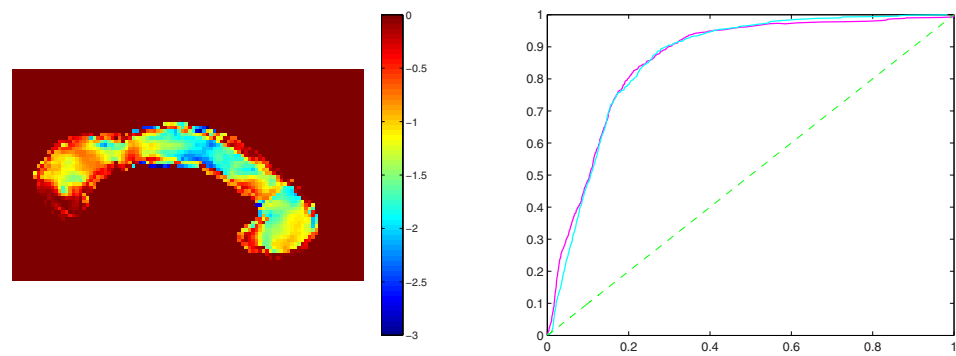

Fig. 1. Left: Voxelwise $p$-values computed from the Hotelling's $T^{2}$ test on the deformation tensors for the average template. The scale shows values of $\log _{10}(p)$. Right: Cumulative distribution of $p$-values $v s$ the corresponding cumulative $p$-value that would be expected from a null distribution for the average shape and the best brain. Pink curve: average brain, blue curve: best individual brain. Dotted line: $x=y$ curve (null distribution). 
registration accuracy and diagnosis that can occur when using an individual brain as a registration target.

\section{Conclusion}

In this paper, we derive a new way to compute mean anatomical templates by minimizing a distance in the space of deformation tensors. The resulting templates may be used for TBM, in which statistical analyses are performed on the deformation tensors mapping individual brains to the target image [14. Because the deformation distance to the template is smaller with a tensor-based mean template, there is a greater chance that intensity-based registrations of individual datasets will not settle in nonglobal minima that are far from the desired correspondence field. In neuroscientific studies, this could be helpful in detecting anatomical differences, for instance in groups of individuals with neurodegenerative diseases, or in designs where the power of treatment to counteract degeneration is evaluated. Two caveats are necessary regarding the interpretation of this data. First, strictly speaking we do not have ground truth regarding the extent and degree of atrophy or neurodegeneration in HIV/AIDS. So, although an approach that finds greater disease effect sizes is likely to be more accurate than one that fails to detect disease, it would be better to compare these models in a predictive design where ground truth regarding the dependent measure is known (i.e., morphometry predicting cognitive scores or future atrophic change). Second, it may be more appropriate to use the mean shape anatomical template derived here in conjunction with registration algorithms whose cost functions are explicitly based on the log-transformed deformation tensors, such as those found for instance in 4 and [19. To do this, we are working on a unified registration and statistical analysis framework in which the regularizer, mean template, and voxel-based statistical analysis are all based on the same log-Euclidean metric.

\section{References}

1. Arsigny, V., et al.: Log-Euclidean metrics for fast and simple calculus on diffusion tensors. Mag. Res. in Med. 56, 411-421 (2006)

2. Beg, M.F., et al.: Computing large deformation metric mappings via geodesic flow on diffeomorphisms. Int. J. of Comp. Vision 61, 139-157 (2005)

3. Bro-Nielsen, M., Gramkow, C.: Fast fluid registration of medical images. Visualization in Biomedical Computing, 267-276 (1996)

4. Brun, C., et al.: Comparison of Standard and Riemannian Elasticity for TensorBased Morphometry in HIV/AIDS. In: MICCAI workshop on Statistical Registration: Pair-wise and Group-wise Alignment and Atlas Formation (submitted, 2007)

5. Chiang, M.C., et al.: 3D pattern of brain atrophy in HIV/AIDS visualized using tensor-based morphometry. Neuroimage 34, 44-60 (2007)

6. Christensen, G.E., et al.: Deformable templates using large deformation kinematics. IEEE-TIP 5, 1435-1447 (1996)

7. Gerig, G., et al.: Computational anatomy to assess longitudinal trajectory of the brain. In: 3DPVT, pp. 1041-1047 (2006) 
8. Gramkow, C.: Registration of 2D and 3D medical images, Master's thesis, Danish Technical University, Copenhagen, Denmark (1996)

9. Guimond, et al.: Average brain models: a convergence study. Comp. Vis. and Im. Understanding 77, 192-210 (1999)

10. Kochunov, P., et al.: An optimized individual target brain in the Talairach coordinate system. Neuroimage 17, 922-927 (2003)

11. Kochunov, P., et al.: Regional spatial normalization: toward an optimal target. J. Comp. Assist. Tomogr. 25, 805-816 (2001)

12. Kochunov, P., et al.: Mapping structural differences of the corpus callosum in individuals with 18q deletions using targetless regional spatial normalization. Hum. Brain Map. 24, 325-331 (2005)

13. Leow, A.D., et al.: Statistical properties of Jacobian maps and inverse-consistent deformations in non- linear image registration. IEEE-TMI 26, 822-832 (2007)

14. Leporé, N., et al.: Multivariate Statistics of the Jacobian Matrices in Tensor-Based Morphometry and their application to HIV/AIDS. In: Larsen, R., Nielsen, M., Sporring, J. (eds.) MICCAI 2006. LNCS, vol. 4190, Springer, Heidelberg (2006)

15. Lorenzen, P., et al.: Multi-class Posterior Atlas Formation via Unbiased KullbackLeibler Template Estimation. In: Barillot, C., Haynor, D.R., Hellier, P. (eds.) MICCAI 2004. LNCS, vol. 3216, pp. 95-102. Springer, Heidelberg (2004)

16. Lorenzen, P., et al.: Multi-modal image set registration and atlas formation. Med. Imag. Analysis 10, 440-451 (2006)

17. Miller, M.I.: Computational anatomy: shape, growth and atrophy comparison via diffeomorphisms. Neuroimage 23(Suppl. 1), 19-33 (2004)

18. Nichols, T.E., Holmes, A.P.: Non parametric permutation tests for functional neuroimaging: a primer with examples. Hum. Brain Map. 15, 1-25 (2001)

19. Pennec, X., et al.: Riemannian elasticity: A statistical regularization framework for non-linear registration. In: Duncan, J.S., Gerig, G. (eds.) MICCAI 2005. LNCS, vol. 3749, pp. 943-950. Springer, Heidelberg (2005)

20. Pennec, X.: Left-invariant Riemannian elasticity: a distance on shape diffeomorphisms? In: MFCA, pp. 1-13 (2006)

21. Studholme, C., et al.: Detecting spatially consistent structural differences in Alzheimer's and fronto-temporal dementia using deformation morphometry. In: Niessen, W.J., Viergever, M.A. (eds.) MICCAI 2001. LNCS, vol. 2208, pp. 41-48. Springer, Heidelberg (2001)

22. Studholme, C., Cardenas, V.: A template free approach to volumetric spatial normalization of brain anatomy. Patt. Recogn. Lett. 25, 1191-1202 (2004)

23. Thompson, P.M., et al.: Growth Patterns in the Developing Brain Detected By Using Continuum-Mechanical Tensor Maps. Nature 404, 190-193 (2000)

24. Thompson, P.M., et al.: Mathematical/Computational Challenges in Creating Population-Based Brain Atlases. Hum. Brain Map. 9, 81-89 (2000)

25. Thompson, P.M., et al.: Thinning of the cerebral cortex visualized in HIV/AIDS reflects CD4+ T-lymphocyte decline. Proc. Nat. Acad. Sci. 102, 15647-15652 (2005)

26. Thompson, P.M., et al.: 3D mapping of ventricular and corpus callosum abnormalities in HIV/AIDS. Neuroimage 31, 12-23 (2006)

27. Twining, C.J.: A unified information-theoretic approach to groupwise non-rigid registration and model building. In: Duncan, J.S., Gerig, G. (eds.) MICCAI 2005. LNCS, vol. 3749, pp. 190-193. Springer, Heidelberg (2005)

28. Woods, R.P.: Characterizing volume and surface deformation in an atlas framework: theory, applications and implementation. Neuroimage 18, 769-788 (2003)

29. Younes, L.: Jacobi fields in groups of diffeomorphisms and applications. Quar. J. of Appl. Math 65, 113-134 (2007) 